\title{
A systems approach to shape and topology optimisation of mechanical structures
}

\author{
K. Fiedler, B. F. Rolfe, A. Asgari \& T. de Souza \\ Institute for Frontier Materials, Deakin University, Australia
}

\begin{abstract}
Optimisation techniques have become more and more important as the possibility of simulating complex mechanical structures has become a reality. A common tool in the layout design of structural parts is the topology optimisation method, which finds an optimum material distribution within a given geometrical design space to best meet loading conditions and constraints.

Another important method is shape optimisation, which optimises weight given parametric geometric constraints. In the case of complex shaped parts or elaborate assemblies, for example automobile body structures, shape optimisation is still hard to do; mainly due to the difficulty in translating shape design parameters into meaningful analysis models. Tools like the parametric geometry package SFE CONCEPT are designed to mitigate these issues.

Nevertheless, shape methods usually cannot suggest new load path configurations, while topology methods are often confined to single parts. To overcome these limitations the authors have developed a method that combines both approaches into an Integral Shape/Topology Method (IST) that is capable of finding new optimal solutions.

This is achieved by an automated optimisation loop and can be applied for both thin walled structures as well as solid 3D geometries. When optimising structures by applying IST, global optimum solutions can be determined that may not be obtained with isolated shape- or topology-optimisation methods.

Keywords: topology optimisation, shape optimisation, simultaneous shape and topology optimisation, structural optimisation, IST.
\end{abstract}




\section{Introduction}

In structural mechanics, the requirements for structural parts are highly complex. And yet, in a highly competitive environment, engineers are expected to shorten development and test time, while also cutting material and production costs. Moreover, energy efficiency plays an increasingly important role in the life cycle of any product. One way to achieve this is light-weighting of structures, where the engineers aim to minimize material usage and simultaneously maximize performance. In the transportation industry, light-weighting is a key enabler for reducing fuel consumption and $\mathrm{CO}_{2}$ emission. In this context, simulation and optimisation methods are of crucial importance.

Today, in designing load bearing parts, the engineer often develops a structural layout using topology optimisation (Bendsøe and Kikuchi [1], Suzuki and Kikuchi [2]). The necessary next step is then to either fine-tune the resulting shape or else translate it into multiple manufacturable parts.

In this context, shape optimisation is limited to fine-tuning the topology optimisation result with respect to local details, e.g. curvature and local stresses.

Put simply, topology optimisation and shape optimisation today are still two distinct fields. While shape optimisation approaches in general are incapable of varying topological layouts, topology methods either use shape design for finetuning only, or else cannot account for specific characteristics of the parts. For example, thin walled structures or complex relationships between members. On the other hand, it may be beneficial to simultaneously do large scale shape variation to find an optimum shape with corresponding optimum load path layout.

The authors have developed a method that can tackle both of these aspects at the same time.

Figure 1 illustrates a traditional process flow whereas Figure 2 shows the IST approach where shape variation is an integral part of a combined optimisation process.

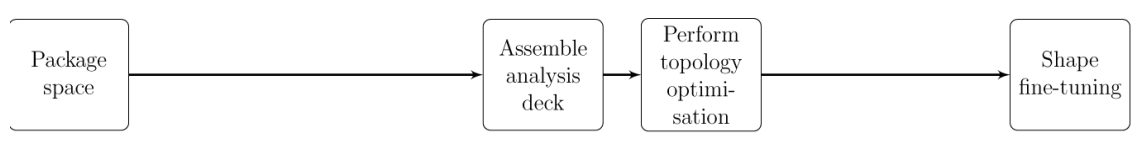

Figure 1: Traditional topology optimisation method.

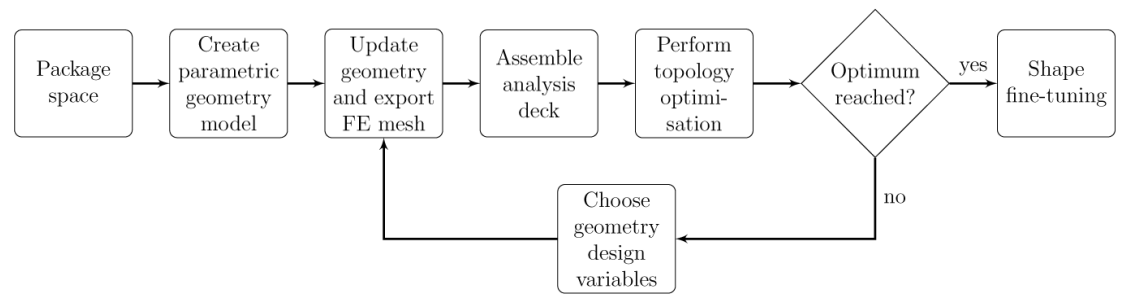

Figure 2: Integral Shape/Topology Method (IST). 


\subsection{Review of existing approaches}

A number of combined shape and topology methods have been proposed and published, and will be briefly discussed in this section.

In the method described by Balamurugan et al. [3], shape modifications are implicitly applied using B-splines and essentially accelerate the topology optimisation algorithm without delivering qualitatively different results compared to a pure topology optimisation approach. A similar idea has been described by Cappello and Mancuso [4], where shape modification is applied on the topology optimisation result in order to smooth rough boundaries. Their approach is limited to two dimensions, and does not allow the definition of geometric design variables.

Ansola et al. [5] adopts a unique approach by starting with a plane finite element mesh in two dimensions. The mesh-topology stays constant (i.e. the connectivity of elements relative to each other cannot change), but grids are allowed to move perpendicular to a fixed plane. Simultaneously, a density distribution for the finite element is determined. The algorithm is limited to sheet structures consisting of a single part, and can deliver only limited out of plane shapes.

In some ways IST is similar to the application of large scale variation as described by Volz et al. [6]. Here the authors employ SFE CONCEPT as the tool of choice to create parametric geometry for an automobile body structure. However, the topology optimisation described is not based on a density method. Rather, predefined structural members are either added or subtracted from the structure.

IST is novel in the sense that large scale shape variation can be described explicitly using intuitive structurally meaningful geometry parameters combined with the established topology optimisation algorithms. This allows for a much wider range of applications than existing hybrid methods.

\subsection{Aim}

In this paper we introduce the basic principles of IST, point out a number of issues that arise with variable geometry combined with a topology optimisation procedure, and provide an example of a combined shape/topology optimisation of a mechanical component.

\section{Process description}

In order for an automated optimisation loop to work, variation in geometric characteristics need to be created in an automated batch process. Here the parametric CAD tool SFE CONCEPT v4.2 was chosen. SFE CONCEPT enables the creation of parametric geometry with the capacity to do large scale shape modification that can be controlled in a batch loop (Zimmer et al. [7]). Hereby, intuitive shape design variables can be defined and result in consistent complex geometrical variation (Duddeck et al. [8]), while the integrated meshing tool generates a finite element (FE) model. 
For the topology optimisation, the Solid Isotropic Material with Penalty (SIMP) method (Rozvany [9]) both for shell and solid elements was used, as provided by the Altair Hyperworks software package Optistruct v11 (Schramm and Zhou [10]).

A number of scripts and tools developed in $\mathrm{C}++$ and Perl combine the commercial software packages into a closed process loop, as shown in Figure 2.

For a set of geometric design variables $d v_{1}, \ldots, d v_{n}$, the parametric model is updated and a FE mesh generated. Loads and constraints are then applied, and a topology optimisation is performed. A subsequent topology optimisation finds optimum load path layouts.

\subsection{Flexible and dynamic analysis model setup}

IST performs these steps in an automated batch loop with a highly flexible control mechanism. For example, boundary conditions can be created dynamically, and check functions can be defined via plug-ins. Also, the handling and extraction of analysis results is managed.

In the example provided in Section 3 later in the paper we utilize IST to vary the initial package space for a subsequent topology optimisation procedure. This adds an additional dimension to the search space for a minimum mass solution and allows us to consider an additional manufacturing constraint.

\subsection{Problems associated with varying geometry}

Performing optimisation of any system requires a structured approach. Design variables need to be selected and a single real-valued objective function must be defined. When using a SIMP algorithm to generate load path concepts, two main approaches to establish an objective function prevail:

1. Minimize mass under some performance constraint (typically displacements, stresses, Eigenfrequencies, and manufacturing considerations)

2. Maximize performance for a fixed volume or mass fraction $\delta$, allowing the SIMP algorithm to take away a defined volume/mass of material equal to $1-\delta$ times the initial volume/mass.

In the first case, the algorithm will determine a material distribution with minimum mass, whereby the amount of remaining material is a priori unknown. In the second case, the amount of material (mass or volume) to be used is fixed, and a load path distribution is generated such that the performance of the structure is maximized.

Using IST, a problem associated with varying initial geometries needs to be addressed: When we generate a number of different geometries, and subsequently take away material by applying topology optimisation, the resulting structures may have different masses and different performance results, which makes it difficult to compare one sample with another. In this section we explore a number of possibilities to solve this problem. 


\subsection{Topology optimisation minimizing mass under performance constraints}

For structures where the mass is minimized under some performance constraints, we have a single-objective problem, and topology optimisation results for different initial geometries are comparable. The optimisation of the shape and load paths of a brake pedal in Section 3 is an example of this approach.

\subsection{Topology optimisation maximizing performance under volume fraction constraint}

For structures where the objective function is some performance figure, we need to find a solution that allows us to directly compare topology optimisation results for varying initial geometries. Let's consider a structure that consists of nondesignable parts (i.e. regions that are not subject to SIMP), and a single designable component, with some volume fraction constraint $\delta$. With the naming conventions

$$
\begin{aligned}
m_{\mathrm{nd}} & =\text { Mass of the non-designable region } \\
m_{\mathrm{d}} & =\text { Mass of the designable region } \\
m_{\mathrm{init}} & =m_{\mathrm{nd}}+m_{\mathrm{d}}=\text { Initial mass before topology optimisation } \\
\delta & =\text { Mass fraction for the topology optimisation }
\end{aligned}
$$

the resulting mass after a topology optimisation run will be

$$
m_{\mathrm{res}}=m_{\mathrm{nd}}+\delta m_{\mathrm{d}}
$$

Since the initial geometry is allowed to change, masses $m_{\mathrm{init}, i}, m_{\mathrm{init}, j}$ for two geometrically different designs $i \neq j$ vary, such that in general

$$
m_{\mathrm{res}, i} \neq m_{\mathrm{res}, j} \text {. }
$$

Performance results for these two designs in general will be different, and the two designs are not directly comparable anymore. There are three ways to solve this issue:

The first approach is to do a multi-objective optimisation, where mass and performance are simultaneously assessed. This leads to a Pareto frontier of feasible designs, that need to be interpreted and evaluated by a human operator.

The second approach is to make sure that the resulting mass for the different initial geometries is constant by adjusting the volume fraction for the SIMP algorithm accordingly: Choose $\delta_{i}$ for each initial geometry such that

$$
m_{\mathrm{res}}=m_{\mathrm{nd}, i}+\delta_{i} m_{\mathrm{d}, i}=\mathrm{constant}, \quad \text { or } \quad \delta_{i}=\frac{m_{\mathrm{res}}-m_{\mathrm{nd}, i}}{m_{\mathrm{d}, i}} .
$$

Thus, for a lighter initial geometry with mass $m_{\text {init }, i}$ the volume fraction value $\delta_{i}$ needs to be bigger than for a geometry with more mass $m_{\text {init }, j}$. After topology 
optimisation this leads to structures with identical mass. The problem with this is that varying mass fractions can lead to qualitatively different results.

Finally, we can constrain the shape design parameters to assure that the resulting masses are constant. Assuming a fixed volume fraction $\delta$, choose the shape design variables $d v_{1}, \ldots, d v_{n}$ such that

$$
m_{\mathrm{nd}, i}+\delta m_{\mathrm{d}, i}=m_{\mathrm{nd}, j}+\delta m_{\mathrm{d}, j}
$$

for all geometrically different designs $i, j$, resulting in $m_{\mathrm{res}, i}=m_{\mathrm{res}, j}$.

In this paper these options will not be discussed in detail; here we only show examples where topology optimisation is performed under a displacement constraint.

\section{Example: variable initial package space for topology optimisation}

In this section we apply IST to a simple problem, where a brake pedal is to be designed using the least amount of material necessary. As an additional constraint we require the component be constructed from a light weight aluminium extruded profile in order to reduce manufacturing costs compared to a cast structure.

\subsection{Problem setup}

Figure 3(a) shows the basic geometry and loads in side view. The rotation axis $\mathbf{R}$ is fixed, allowing for rotation around the $y$-axis only. The attachment point for the brake cylinder $\mathbf{A}$ is constrained in $\mathbf{x}$-direction. A force $\mathbf{F}$ of $1000 \mathrm{~N}$ is applied at

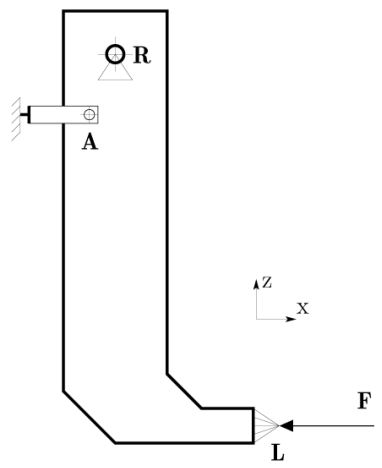

(a) Schematic layout in side view with boundary conditions

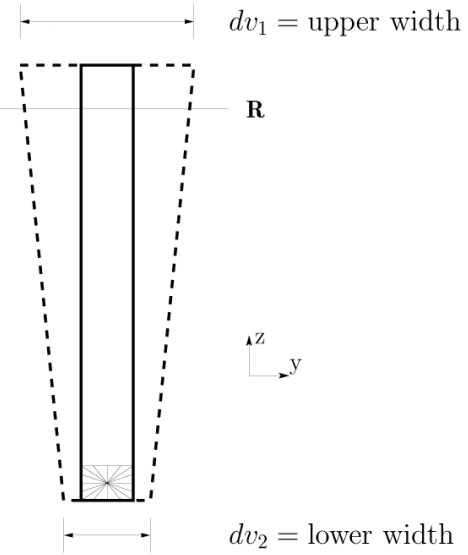

(b) Geometry design variables in front view

Figure 3: Brake pedal: layout and variable geometry. 
point $\mathbf{L}$ in negative $\mathbf{x}$-direction, while the contact point $\mathbf{L}$ is allowed a maximum displacement of $0.5 \mathrm{~mm}$.

\subsection{Variable geometrical design space}

By varying the upper and lower width of the blank, the initial package space is allowed to change, as indicated in the front view in Figure 3(b). This variability results in a wedge shaped initial geometry which is then subjected to SIMP.

The operation sequence is as described in Figure 2. Values for the two geometry design variables (upper and lower width) are chosen, then the parametric SFE CONCEPT geometry model is updated and a finite element mesh created. Then boundary conditions are applied, and a topology optimisation is performed.

\subsection{Design of experiments}

The objective of the topology optimisations is to minimize mass given two constraints: a displacement constraint, and an extrusion constraint in y-direction. A design of experiments (DOE) with a coarse mesh (6 mm HEXA voxel mesh) has been conducted with:

$$
\begin{aligned}
& d v_{1}=\text { upper width }=6,8, \ldots, 20 \mathrm{~mm} \\
& d v_{2}=\text { lower width }=6,8, \ldots, 12 \mathrm{~mm},
\end{aligned}
$$

which results in the response surface shown in Figure 4(a). For different initial geometries we get distinct structural load path layouts, and it can be seen that the best results (meaning lowest mass results) are achieved when the lower width is at its lower limit of $d v_{2}=6 \mathrm{~mm}$.

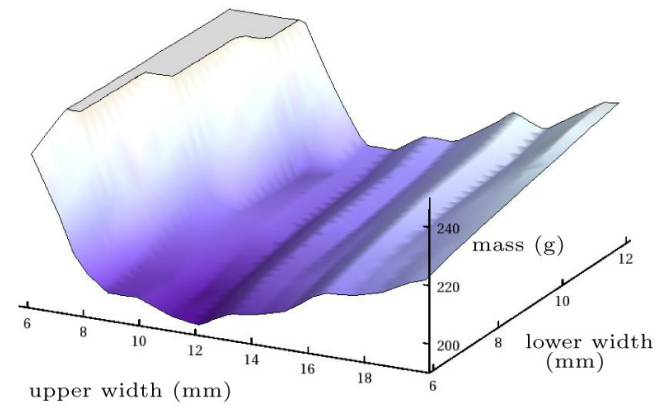

(a) 3D plot

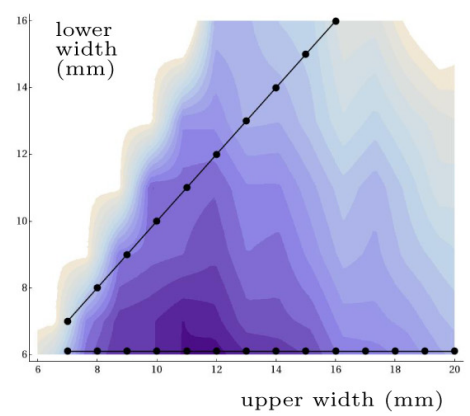

(b) Contour plot

Figure 4: Resulting mass after topology optimisation. 


\subsection{Results}

For a refined DOE study we therefore assume $d v_{2}=$ constant $=6 \mathrm{~mm}$, while varying $d v_{1}$ between $6 \mathrm{~mm}$ and $20 \mathrm{~mm}$. For this study a finer mesh size for the voxel elements of $2 \mathrm{~mm}$ was used. Figure 5(a) shows the resulting masses, with the lowest mass being $192 \mathrm{~g}$ for the design at $d v_{1}=12$. The graph corresponds to the horizontal dotted line in Figure 4(b). The corresponding wedge-shaped structure is shown in Figure 6(a) at a mass fraction cut-off value of 0.3.

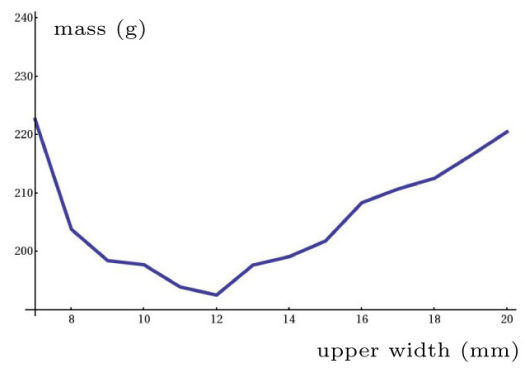

(a) Lower width $=$ constant $=6 \mathrm{~mm}$

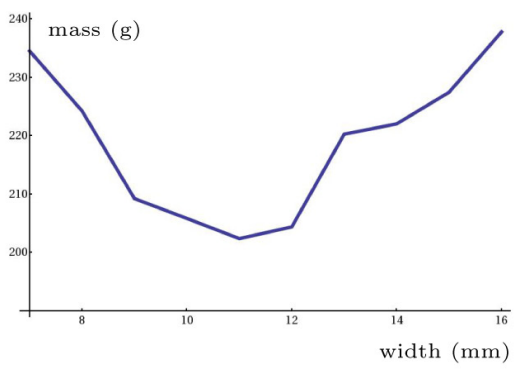

(b) Upper width $=$ lower width

Figure 5: Resulting mass for refined DOE.

\subsection{Comparison with a traditional approach}

For validation purposes it is necessary to compare the combined shape/topology (IST) technique to a conventional approach where the designer would start with a flat blank and perform a topology optimisation to find a suitable load path concept.

It is by no means evident that the engineer would choose the 'right' width to start with. For an initial blank thickness of $12 \mathrm{~mm}$, topology optimisation returns a structure weighing $206 \mathrm{~g}$, while the minimum mass for a constant width blank is at $11 \mathrm{~mm}$ width (202 g). Figure 6(b) shows the topology optimisation result for a constant blank width of $11 \mathrm{~mm}$. The masses after topology optimisation for different material thicknesses are shown in Figure 5(b), corresponding with the diagonal dotted line in Figure 4(b).

Therefore, even if the optimum width was chosen, the resulting design would still be $5 \%$ heavier than the results achieved when applying IST.

In this example, the IST approach essentially added an additional manufacturing constraint to the topology optimisation method: Where traditionally the package space for a topology optimisation would be fixed, the manufacturing restriction to extruded profiles made it beneficial to incorporate variable initial design space characteristics. Thus it was possible to derive an optimum angle for the wedge shaped brake pedal. 


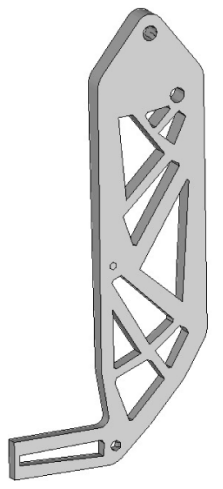

(a) Wedge shaped; upper width = $12 \mathrm{~mm}$, lower width $=6 \mathrm{~mm}$

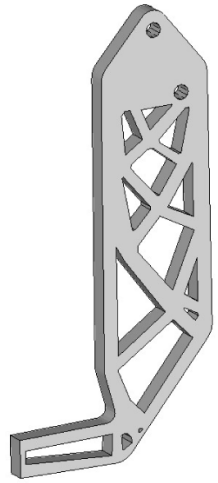

(b) Constant thickness; width = $11 \mathrm{~mm}$

Figure 6: Topology optimisation results.

\section{Conclusion}

We have introduced the novel Integral Shape and Topology (IST) optimisation method which allows simultaneous large scale shape variation and load path layout optimisation. This method has been shown to be useful for a voxel-based solid structure, where it:

- leads to lower mass structures

- adds additional manufacturing constraints to topology optimisation algorithms

In the example discussed, the optimum found by IST could not have been found by traditional topology optimisation methods alone, and IST allowed us to cater for the specific manufacturing requirement, i.e. the part being made from an extruded profile. A mass saving of $5 \%$ over a conventional topology optimisation approach was achieved.

\section{Acknowledgements}

The authors would like to thank SFE GmbH, Berlin, for providing the software package SFE CONCEPT, and the Automotive Technology Cooperative Research Centre (AutoCRC) project C2-23 for their financial support.

\section{References}

[1] Bendsøe, M. and Kikuchi, N., Generating optimal topologies in structural design using a homogenization method. Computer Methods in Applied Mechanics and Engineering, 71(2), pp. 197-224, 1988. 
[2] Suzuki, K. and Kikuchi, N., A homogenization method for shape and topology optimization. Computer Methods in Applied Mechanics and Engineering, 93(3), pp. 291-318, 1991.

[3] Balamurugan, R., Ramakrishnan, C.V. and Swaminathan, N., Integrated optimal design of structures under multiple loads for topology and shape using genetic algorithm. Engineering Computations, 23(1/2), pp. 57-83, 2006.

[4] Cappello, F. and Mancuso, A., A genetic algorithm for combined topology and shape optimisations. Computer-Aided Design, 35(8), pp. 761-769, 2003.

[5] Ansola, R., Canales, J., Tarrago, J.A. and Rasmussen, J., An integrated approach for shape and topology optimization of shell structures. Computers \& Structures, 80(5-6), pp. 449-458, 2002.

[6] Volz, K., Frodl, B., Dirschmid, F., Stryczek, R. and Zimmer, H., Optimizing topology and shape for crashworthiness in vehicle product development. International Automotive Body Congress (IABC), 2007.

[7] Zimmer, H., Prabhuwaingankar, M. and Duddeck, F., Topology \& geometry based structure optimization using implicit parametric models and ls-opt. $7^{\text {th }}$ European LS-DYNA Conference, 2009.

[8] Duddeck, F., Hunkeler, S., Zimmer, H., Rota, L. and Zarroug, M., Shape optimization for crashworthiness. Weimar Optimization and Stochastic Days, 2009.

[9] Rozvany, G.I.N., Aims, scope, methods, history and unified terminology of computer-aided topology optimization in structural mechanics. Structural and Multidisciplinary Optimization, 21(2), pp. 90-108, 2001.

[10] Schramm, U. and Zhou, M., Recent Developments in the Commercial Implementation of Topology Optimization, Springer Netherlands, volume 137 of Solid Mechanics and Its Applications, pp. 239-248, 2006. 